\title{
Efficacy of bowel preparation regimens for colon capsule endoscopy: a systematic review and meta-analysis
}

\section{(ㄷ)(1) $९$}

\section{Authors}

Thomas Bjoersum-Meyer ${ }^{1}$, Karolina Skonieczna-Zydecka², Pablo Cortegoso Valdivia ${ }^{3}$, Irene Stenfors ${ }^{4}$, Ivan Lyutakov ${ }^{5}$, Emanuele Rondonotti ${ }^{6}$, Marco Pennazio ${ }^{7}$, Wojciech Marlicz ${ }^{8,9}$, Gunnar Baatrup ${ }^{1}$, Anastasios Koulaouzidis ${ }^{10}$, Ervin Toth $^{4}$

Institutions

1 Department of Surgery, Odense University Hospital, Odense Denmark

2 Department of Biochemical Sciences, Pomeranian Medical University, Szczecin, Poland

3 Gastroenterology and Endoscopy Unit, University Hospital of Parma, University of Parma, Parma, Italy.

4 Department of Gastroenterology, Skåne University Hospital, Malmö, Lund University, Sweden

5 Department of Gastroenterology, University Hospital “Tsaritsa Yoanna - ISUL”, Medical University Sofia, Bulgaria

6 Gastroenterology Unit, Valduce Hospital, Como, Italy

7 University Division of Gastroenterology, City of Health and Science University Hospital, Turin, Italy

8 Department of Gastroenterology, Pomeranian Medical University, Szczecin, Poland

9 The Centre for Digestive Diseases, Endoklinika, Szczecin, Poland

10 Department of Social Medicine \& Public Health, Pomeranian Medical University, Szczecin, Poland

submitted 25.2.2021

accepted after revision $\quad 4.6 .2021$

\section{Bibliography}

Endosc Int Open 2021; 09: E1658-E1673

DOI 10.1055/a-1529-5814

ISSN 2364-3722

\section{(C) 2021. The Author(s).}

This is an open access article published by Thieme under the terms of the Creative Commons Attribution-NonDerivative-NonCommercial License, permitting copying and reproduction so long as the original work is given appropriate credit. Contents may not be used for commercial purposes, or adapted, remixed, transformed or built upon. (https://creativecommons.org/licenses/by-nc-nd/4.0/)

Georg Thieme Verlag KG, Rüdigerstraße 14,

70469 Stuttgart, Germany

Corresponding author

Thomas Bjoersum-Meyer, Department of Surgery, Odense University Hospital, J.B. Winsløwsvej 4, Odense C, 5000,

Denmark

Thomas.bjoersum-meyer@rsyd.dk $\circledast$ Supplementary material is available under https://doi.org/10.1055/a-1529-5814

\section{ABSTRACT}

Background and study aims Colon capsule endoscopy (CCE) is an alternative to conventional colonoscopy (CC) in specific clinical settings. High completion rates (CRs) and adequate cleanliness rates (ACRs) are fundamental quality parameters if CCE is to be widely implemented as a CC equivalent diagnostic modality. We conducted a systematic review and meta-analysis to investigate the efficacy of different bowel preparations regimens on CR and ACR in CCE. Patients and methods We performed a systematic literature search in PubMed, Embase, CINAHL, Web of Science, and the Cochrane Library. Data were independently extracted per the Preferred Reporting Items for Systematic Reviews and Meta-Analyses (PRISMA). The primary outcome measures ( $C R, A C R)$ were retrieved from the individual studies and pooled event rates were calculated.

Results Thirty-four observational (OBS) studies $(n=3,789)$ and 12 randomized clinical trials (RCTs) $(n=1,214)$ comprising a total 5,003 patients were included. The overall CR was $0.798(95 \% \mathrm{Cl}, 0.764-0.828)$; the highest CRs were observed with sodium phosphate $(\mathrm{NaP})+$ gastrografin booster $(n=2, C R=0.931,95 \% \mathrm{Cl}, 0.820-0.976)$. The overall ACR was $0.768(95 \% \mathrm{Cl}, 0.735-0.797)$; the highest $A C R s$ were observed with polyethylene glycol (PEG)+ magnesium citrate $(n=4, E R=0.953,95 \% \mathrm{Cl}, 0.896-0.979)$.

Conclusions In the largest meta-analysis on CCE bowel preparation regimens, we found that both CRs and ACRs are suboptimal compared to the minimum recommended standards for CC. PEG laxative and NaP booster were the most commonly used but were not associated with higher CRs or ACRs. Well-designed studies on CCE should be performed to find the optimal preparation regimen. 


\section{Introduction}

Colon capsule endoscopy (CCE) was introduced in 2006 as a novel diagnostic modality for colorectal examination [1]. In contrast to conventional colonoscopy (CC), CCE does not have capabilities for biopsy, polypectomy or lens cleansing and provides a lower-quality image (especially when compared to the newer generation colonoscopes); however, it offers a comfortable, minimally invasive, diagnostic procedure with a favorable safety profile and no need for sedation [2]. A recent systematic review showed that CCE has high sensitivity and specificity for per-patient polyp detection, compared to CC [3]. Nevertheless, improvements in colon cleanliness and the rate of complete examinations (to mitigate the limitations mentioned above) are needed before wider implementation in screening populations is considered. Studies have consistently shown that improvement in bowel cleanliness increases the sensitivity and specificity of CCE for detecting polyps $>6 \mathrm{~mm}$ [4]. Preparation in CCE is of utmost importance as it allows the detection and better characterization of polypoid findings and other mucosa pathology as well, which otherwise would not have been visible. However, the field of clinical practice in terms of procedural preparation (laxatives, boosters and/or prokinetics) is diverse, and the endoscopic community is keen for guidance based on a critical review of existing literature.

We conducted a comprehensive systematic literature review and meta-analysis to assess the bowel preparation regimens for CCE with specific regard to completion rates (CRs) and adequate cleanliness rates (ACRs). The primary aims were to present pooled estimates for CRs and ACRs stratified by type of laxative, type of booster, and prokinetic. The secondary aimed was to perform a meta-regression analysis for CR, ACR and colon transit times (CTT) with the following explanatory variables: 1) age (mean); 2) sex (\% of males); 3) total number of patients; 4) type of booster; 5) laxative volume with/without a booster; and 6) type of prokinetics.

\section{Patients and methods}

\section{Data sources, search strategy and inclusion/exclusion criteria}

Two independent authors (TBM and IL) searched PubMed, Embase, CINAHL, Web Of Science and the Cochrane library from database inception June 20, 2020 for observational studies (OBS) and randomized controlled trials (RCTs) published in English and reporting CRs, ACRs, and CTTs. The separate arms of RCTs were treated as observational ones, thus included in the pooled estimation, with the indication though of the study design in synthesis plots.

The following search terms were used in PubMed/Embase/ Cochrane Library: ((Colon capsule OR colon capsule endoscopy OR CCE OR pan-enteric capsule OR capsule colonoscopy OR PillCam) AND (bowel preparation OR laxative OR bowel cleansing OR PEG)). The electronic search was supplemented by a manual review of reference lists from eligible publications and relevant reviews.
Inclusion criteria were: (1) peer-reviewed human studies of observational and clinical types; and (2) reporting data on completeness and quality of cleanliness.

We excluded reviews, opinions papers, case reports and conference papers.

\section{Outcomes}

Primary outcomes were CRs and ACRs. The secondary outcome was CTT. We evaluated these outcomes regarding the usage of different types of boosters and prokinetics and different types of laxatives administration. For this analysis, a 4-point scale by Leighton-Rex was modified into 2-point assessment as follows: Adequate = excellent, good; Inadequate = fair, poor [5] .

\section{Data abstraction}

Data on study design and aim, risk of bias (ROB), patient data (e.g., age, sex) and intervention characteristics, (e.g., laxative type, type of laxative administration, type of booster, type of prokinetics) from each study were independently extracted according to the Preferred Reporting Items for Systematic Reviews and Meta-Analyses (PRISMA) standard by four investigators (TB-M, IS, IL, PC) [6].

The extraction list was split in two, and each of them allocated to a pair of investigators who extracted data in duplicates. Whenever data were missing for the review, corresponding authors were contacted for additional information via emails. Disagreements between investigators (TB-M, IS, IL, PC) were resolved through consensus, with the final decision made by two of the senior authors (AK and ET).

\section{Risk of bias in individual studies}

The risk of bias was evaluated by using the STROBE assessment for OBS [7]. Each item from the tool was evaluated and, if fulfilled within the study, 1 point was added. Therefore, the rating was the sum of points received for each item of STROBE. The highest number of points to be achieved was 32 .

The bias for each included RCT was assessed per guidelines of the Cochrane Collaboration's tool [8]. Within each bias category (selection, performance, detection, attrition and reporting) of the assessment tool the level of bias was rated as "low risk", "high risk," or "unclear risk."

Overall, the risk of bias was evaluated based on the assumption that higher number of points in STROBE evaluation in case of OBS and the higher number of low risk-of-bias assessments in RCTs, / the greater quality of a study.

\section{Risk of bias across studies}

To explore the existence of publication or other types of bias, when detected by the inconsistency index $\left(I^{2}\right)$ measuring the proportion of unexplained variation across studies, a funnel plot of standard error by diagnostic yield (DY) was produced.

\section{Data synthesis and statistical analysis}

We conducted a random-effects meta-analysis of outcomes for which $\geq 2$ studies contributed data, using Comprehensive MetaAnalysis V3.3.070 (http://www.meta-analysis.com). The $\mathrm{I}^{2}$ index was applied to measure heterogeneity together with the 
$X^{2}$ test of homogeneity, with $P<0.05$ indicating statistically significant heterogeneity. All analyses tests were two-sided and the significance level was set to 0.05 .

For continuous outcomes, we analyzed the pooled means for endpoint scores using observed cases data. Categorical outcomes were analyzed by calculating the pooled event rates. We conducted subgroup and exploratory maximum likelihood random-effects meta-regression analyses of all outcomes. Finally, we inspected funnel plots and used Egger's regression test to quantify whether publication bias could have influenced the results [9].

Meta-regression variables included: 1 ) age (mean); 2 ) sex (\% of males); 3) total number of patients; 4) type of booster; 5) laxative volume with/without a booster; and 6) type of prokinetics. Finally, we inspected funnel plots and used Egger's regression test and the Duval and Tweedie's trim and fill method if necessary, to quantify whether publication bias could have influenced the results [10]. Z test of the null hypothesis was run. The assumption of homogeneity in effects was tested using the Q statistic with a k-1 degree of freedom (k-the number of studies). In addition, we used the index of heterogeneity - Higgins' $\mathrm{I}^{2}$ - and assumed that heterogeneity is considered high if $\mathrm{I}^{2}$ $>75 \%$ ). To confirm the robustness of the results, we performed a sensitivity analysis, i. e. a leave-one-out approach.

\section{Results}

\section{Search results}

The initial search yielded 571 hits. A total of 154 studies were excluded for being duplicates and further 313 studies were omitted after evaluation on the title/abstract level. In the second stage, we subjected 104 full-text articles to review. Of those, 58 articles were excluded, yielding 46 studies that were included in the meta-analysis. A flow diagram of the process of this meta-analysis is shown in $\mathbf{F i g} \mathbf{1}$.

\section{Study, patient and regimen characteristics}

Altogether, 62 study arms with different preparation regimens in 34 OBS studies $(n=3,789)$ and 12 (RCTs) $(n=1,214)$ with a total of 5,003 patients conducted in 15 countries were included in the present meta-analysis. The percentage of men in both study groups was comparable (OBS: $49.46 \%$ vs. RCT: $51.41 \%$ ). The mean age of study participants was 56.39 years (7.66) (OBS: 53.35 [8.77] vs. RCT: 56.45 years [6.22] years). The most commonly used bowel preparation was polyethylene glycol (PEG) (37 studies)/PEG plus ascorbic acid (PEGAA) (22 studies) administrated in a split-dosing regimen (50 studies). The predominantly used boosters were sodium phosphate ( $\mathrm{NaP}$ ) (24 studies) and PEGAA (10 studies). However, regarding NaP usage, most studies utilized non-NaP-based boosters (34 studies).

Prokinetics administered on demand have been added to the protocol for bowel preparation for CCE in all, except five studies, to stimulate the progression of the capsule in the stomach. The most common prokinetics used were domperidone (DOM) (31 studies) and metoclopramide (MET) (11 studies). Patients in all studies included in this analysis received dietary recom-

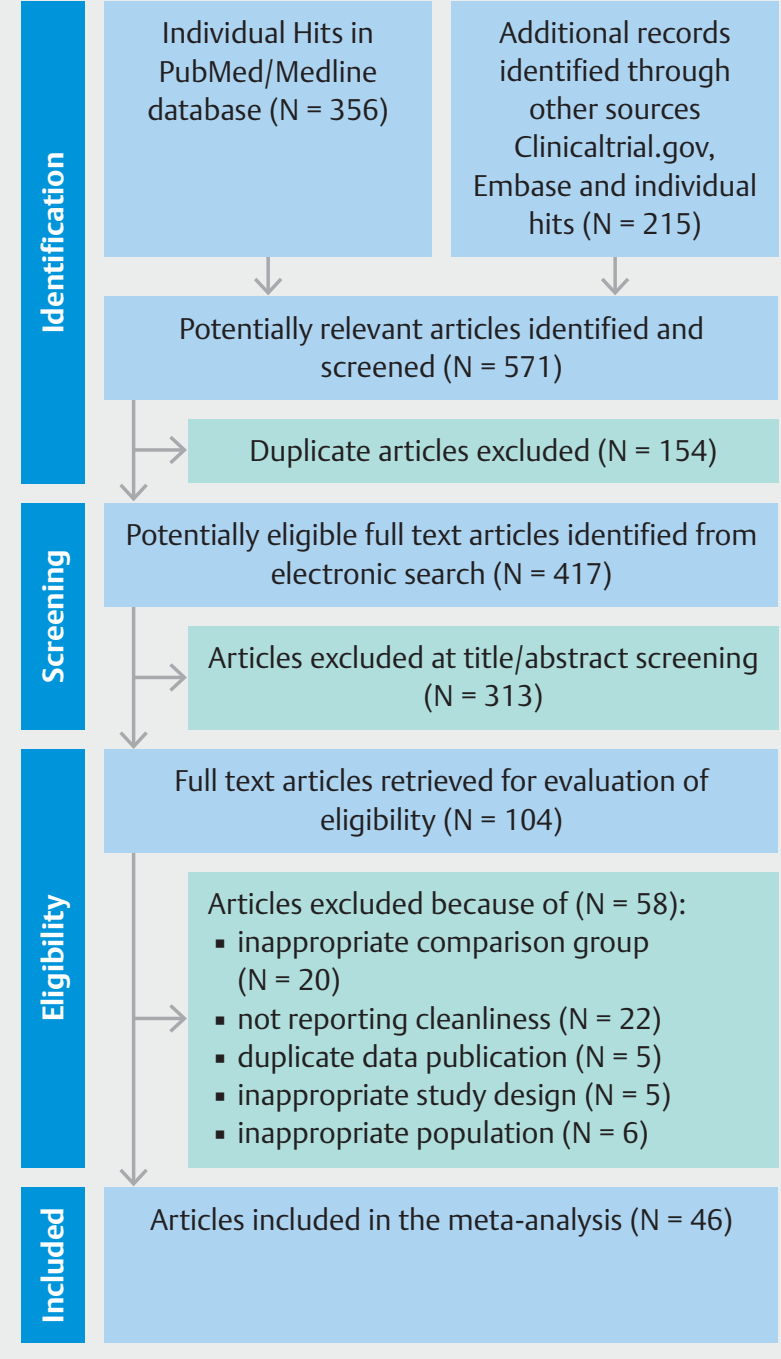

- Fig. 1 Flow diagram of study selection.

mendations before the CCE procedure. Bisacodyl suppositories as a stimulant laxative have been uniformly used on-demand across most studies for stimulating the expulsion of the capsule. The CCE models were either PillCam CCE1 (18 studies) or PillCam CCE2 (44 studies). A pan-enteric Crohn's Capsule (PCC) was used in only one study.

Detailed characteristics of the included studies in this metaanalysis are presented in $>$ Table 1 [11-56].

\section{Risk of bias}

For OBS studies, the mean number of points in STROBE assessment tool was 25.65(5.02) points (median 28, $\min =17$, $\max =$ 32) (Supplementary Table 1 ). The mean number of low risk-ofbias assessments for the RCT studies included in the meta-analysis was 5.83(1.64) points (median=6, $\min =3$, $\max =7$ ) (Supplementary Table 2). There was one OBS study with the highest number of points (32 points) and 2 RCTs with a maximum number of low ROB assessments ( 8 points). None of the includ- 
- Table 1 Study characteristics.

\begin{tabular}{|c|c|c|c|c|c|c|c|c|}
\hline Study & Year & Country & Design & CCE type & $\mathbf{N}$ & Laxative & Booster & Prokinetic(s) \\
\hline Schoofs [11] & 2006 & Belgium & OBS & CCE1 & 41 & PEG & $\mathrm{NaP}$ & DOM \\
\hline van Gossum [12] & 2009 & France & OBS & CCE1 & 332 & PEG & $\mathrm{NaP}$ & DOM \\
\hline Eliakim [13] & 2009 & Israel & OBS & CCE2 & 98 & PEG & $\mathrm{NaP}$ & DOM \\
\hline Gay [14] & 2010 & France & OBS & CCE1 & 128 & PEG & $\mathrm{NaP}$ & DOM \\
\hline Spada [15] & 2011 & Italy & OBS & CCE2 & 117 & PEG & $\mathrm{NaP}$ & DOM \\
\hline $\begin{array}{l}\text { Herrerìas-Gutiérrez } \\
\text { [16] }\end{array}$ & 2011 & Spain & OBS & CCE1 & 144 & PEG & $\mathrm{NaP}$ & DOM \\
\hline Spada [17] & 2011 & Italy & OBS & CCE2 & 47 & PEG & $\mathrm{NaP}$ & DOM \\
\hline Rondonotti [18] & 2014 & Italy & OBS & CCE2 & 50 & PEGAA & $\mathrm{NaP}$ & MET \\
\hline Hagel [19] & 2014 & Germany & OBS & CCE2 & 24 & PEG & $\mathrm{NaP}$ & NA \\
\hline Triantafyllou [20] & 2014 & Greece & OBS & CCE1 & 50 & PEG & $\mathrm{NaP}$ & DOM \\
\hline Saito [21] & 2015 & Japan & OBS & CCE2 & 72 & PEG & $\mathrm{PEG}+\mathrm{MgCit}+\mathrm{MOS}$ & MET/MOS \\
\hline $\operatorname{Rex}[22]$ & 2015 & $\begin{array}{l}\text { USA, France, } \\
\text { China, Spain, } \\
\text { Israel }\end{array}$ & OBS & CCE2 & 792 & PEG & Suprep & MET \\
\hline Spada [23] & 2015 & Italy & OBS & CCE2 & 100 & PEG & $\mathrm{NaP}+\mathrm{GG}$ & DOM \\
\hline Togashi [24] & 2015 & Japan & OBS & CCE2 & 29 & PEGAA & $\mathrm{PEG}+\mathrm{GG}+\mathrm{MOS}$ & DOM \\
\hline Boal Carvalho [25] & 2015 & Portugal & OBS & CCE2 & 12 & PEG & $\mathrm{NaP}$ & DOM \\
\hline $\begin{array}{l}\text { Romero-Vazquez } \\
\text { [26] }\end{array}$ & 2016 & Spain & OBS & $\begin{array}{l}\text { CCE1/ } \\
\text { CCE2 }\end{array}$ & 165 & PEG & $\mathrm{NaP}$ & MET \\
\hline Ota [27] & 2017 & Japan & OBS & CCE2 & 20 & PEG & $\mathrm{PEG}+\mathrm{MgCit}$ & NA \\
\hline Zhou [28] & 2017 & China & OBS & CCE2 & 31 & PEG & $\mathrm{PEG}+\mathrm{MOS}$ & NA \\
\hline Nogales [29] & 2017 & Spain & OBS & CCE2 & 96 & PEG & $\mathrm{NaP}$ & MET \\
\hline Igawa [30] & 2017 & Japan & OBS & CCE2 & 30 & PEG & $\mathrm{PEG}+\mathrm{MgCit}+\mathrm{MOS}$ & MET \\
\hline Sato [31] & 2017 & Japan & OBS & CCE2 & 70 & PEG + MgCit & $\mathrm{PEG}+\mathrm{MgCit}+\mathrm{MOS}$ & MET \\
\hline Pioche [32] & 2018 & France & OBS & CCE2 & 20 & PEG & $\mathrm{NaP}$ & DOM \\
\hline Takano [33] & 2018 & Japan & OBS & CCE2 & 30 & $\begin{array}{l}\text { MgCit+Sen- } \\
\text { noside }\end{array}$ & $\mathrm{PEGAA}+\mathrm{MgCit}$ & MOS \\
\hline Kobaek-Larsen [34] & 2018 & Denmark & OBS & CCE2 & 253 & PEGAA & PEGAA & DOM \\
\hline Okabayashi [35] & 2018 & Japan & OBS & CCE2 & 39 & PEG & $\mathrm{PEG}+\mathrm{CO}$ & MET \\
\hline Eliakim [36] & 2018 & $\begin{array}{l}\text { Israel, Italy, } \\
\text { Spain }\end{array}$ & OBS & PCCE & 41 & PEG & Suprep & MET \\
\hline Parodi [37] & 2018 & Italy, France & OBS & CCE2 & 177 & PEG & $\mathrm{NaP}$ & DOM \\
\hline Baltes [38] & 2018 & Germany & OBS & CCE2 & 36 & PEGAA & $\mathrm{PEGAA}+\mathrm{NaP}$ & DOM \\
\hline Voska [39] & 2019 & $\begin{array}{l}\text { Czech } \\
\text { Republic }\end{array}$ & OBS & CCE2 & 236 & PEG & $\mathrm{NaP}$ & MET \\
\hline Yamada [40] & 2019 & Japan & OBS & CCE2 & 53 & PEG & $\begin{array}{l}\mathrm{PEG}+\mathrm{CO}+\mathrm{NaP}+ \\
\mathrm{MOS}\end{array}$ & NA \\
\hline González-Suárez [41] & 2020 & Spain & OBS & CCE2 & 147 & PEGAA & $P E G A A+G G$ & MET \\
\hline Otani [42] & 2020 & Japan & OBS & CCE2 & 60 & PEG & $\mathrm{PEG}+\mathrm{MgCit}+\mathrm{MOS}$ & NA \\
\hline Pecere [43] & 2020 & Italy & OBS & CCE2 & 222 & PEG & $\mathrm{NaP}+\mathrm{GG}$ & DOM \\
\hline Utano [44] & 2020 & Japan & OBS & CCE2 & 27 & $\begin{array}{l}\text { PEGAA+So- } \\
\text { dium picosul- } \\
\text { fate }\end{array}$ & $\mathrm{MgCit}+\mathrm{GG}$ & DOM \\
\hline
\end{tabular}


- Table 1 (Continuation)

\begin{tabular}{|c|c|c|c|c|c|c|c|c|}
\hline Study & Year & Country & Design & CCE type & $\mathbf{N}$ & Laxative & Booster & Prokinetic(s) \\
\hline Eliakim [45] a & 2006 & Israel & RCT & CCE1 & 44 & PEG & $\mathrm{NaP}$ & Tegaserod \\
\hline Eliakim b & 2006 & & RCT & CCE1 & 46 & PEG & $\mathrm{NaP}$ & Tegaserod \\
\hline Spada [46] a & 2011 & Italy & RCT & CCE2 & 20 & PEG & PEG & DOM \\
\hline Spada b & & & RCT & CCE2 & 20 & PEG & $\mathrm{NaP}$ & DOM \\
\hline Hartmann [47] a & 2012 & Germany & RCT & CCE2 & 26 & PEGAA & PEGAA & DOM \\
\hline Hartmann b & & & RCT & CCE2 & 24 & PEGAA & PEGAA & DOM \\
\hline Kakugawa [48] a & 2012 & Japan & RCT & CCE1 & 33 & PEG & MgCit & MOS \\
\hline Kakugawa b & & & RCT & CCE1 & 31 & PEG & MgCit & MOS \\
\hline Argüelles-Arias [49] a & 2014 & Spain & RCT & CCE2 & 28 & PEGAA & $\mathrm{NaP}$ & DOM \\
\hline Argüelles-Arias b & & & RCT & CCE2 & 30 & PEG & $\mathrm{NaP}$ & DOM \\
\hline Ramos50 a & 2014 & Spain & RCT & CCE1 & 20 & PEG & $\mathrm{NaP}$ & DOM \\
\hline Ramos b & & & RCT & CCE1 & 20 & PEG & $\mathrm{NaP}$ & DOM \\
\hline Brechmann51 a & 2016 & Germany & RCT & CCE1 & 20 & PEGAA & PEGAA & ERY \\
\hline Brechmann b & & & RCT & CCE1 & 6 & PEGAA & PEGAA & ERY \\
\hline Brechmann c & & & RCT & CCE1 & 12 & PEGAA & PEGAA & ERY \\
\hline Brechmann d & & & RCT & CCE1 & 12 & PEGAA & PEGAA + senna tea & ERY \\
\hline Kastenberg [52] a & 2017 & USA, Cyprus & RCT & CCE2 & 55 & PEG & Suprep+GG & MET/ERY \\
\hline Kastenberg b & & & RCT & CCE2 & 52 & PEG & Suprep & MET/ERY \\
\hline Alvarez-Urturi [53] a & 2017 & Spain & RCT & CCE1 & 27 & PEGAA & PEGAA & MET \\
\hline Alvarez-Urturi b & & & RCT & CCE1 & 24 & PEGAA & $\mathrm{NaP}$ & MET \\
\hline Buijs [54] a & 2018 & Denmark & RCT & CCE2 & 57 & PEGAA & $\begin{array}{l}\text { PEGAA + chewing } \\
\text { gum }\end{array}$ & DOM \\
\hline Buijs b & & & RCT & CCE2 & 48 & PEGAA & PEGAA + coffee & DOM \\
\hline Buijs C & & & RCT & CCE2 & 60 & PEGAA & PEGAA & DOM \\
\hline Kroijer [55] a & 2018 & Denmark & RCT & CCE2 & 60 & PEGAA & PEGAA & DOM \\
\hline Kroijer b & & & RCT & CCE2 & 60 & PEGAA & $\begin{array}{l}\text { Sulfate based solu- } \\
\text { tion }\end{array}$ & DOM \\
\hline Kroijer c & & & RCT & CCE2 & 60 & PEGAA & $P E G A A+G G$ & DOM \\
\hline Ohmiya [56] a & 2018 & Japan & RCT & CCE2 & 152 & PEGAA & $\mathrm{PEGAA}+\mathrm{CO}$ & $\begin{array}{l}\text { MET or MgCit + } \\
\text { MOS }\end{array}$ \\
\hline Ohmiya b & & & RCT & CCE2 & 167 & PEGAA & PEGAA & MOS \\
\hline
\end{tabular}

ed studies describes methods to address the risk of bias within their observations.

\section{Regimen effects on CCE completion rate}

Using a random-effects model, the overall pooled CCE CR was $0.798(95 \% \mathrm{Cl}, 0.764-0.828)$. Between-study heterogeneity was present for $C R$ by booster, laxative type, and prokinetic type $\left(I^{2}=96.25, P=0.000\right)$. The most commonly used booster in the included studies was $\mathrm{NaP}(n=24)$; pooled $C R$ of 0.830
( $95 \% \mathrm{Cl}, 0.781-0.871)$ ( $\mathbf{F i g . 2}$ ). The highest completion rate was observed in studies $(\mathrm{n}=2)$ where $\mathrm{NaP}$ was used in combination with gastrografin (GG) $(\mathrm{CR}=0.931,95 \% \mathrm{Cl}, 0.820-0.976)$. The CR for the most widely used laxative (PEG) was 0.822 (95\% $\mathrm{Cl}, 0.785-0.854)(\vee$ Fig. 3). The overall CR for studies using prokinetics was $0.798(96 \% \mathrm{Cl}, 0.724-0.854)$ ( $\triangleright$ Fig.4). A higher, but not statistically significant, CR was observed for studies in which prokinetic was administered $(\mathrm{CR}=0.801,95 \% \mathrm{Cl}, 0.765$ $0.832)$ as compared to studies not using a prokinetic $(C R=$ 


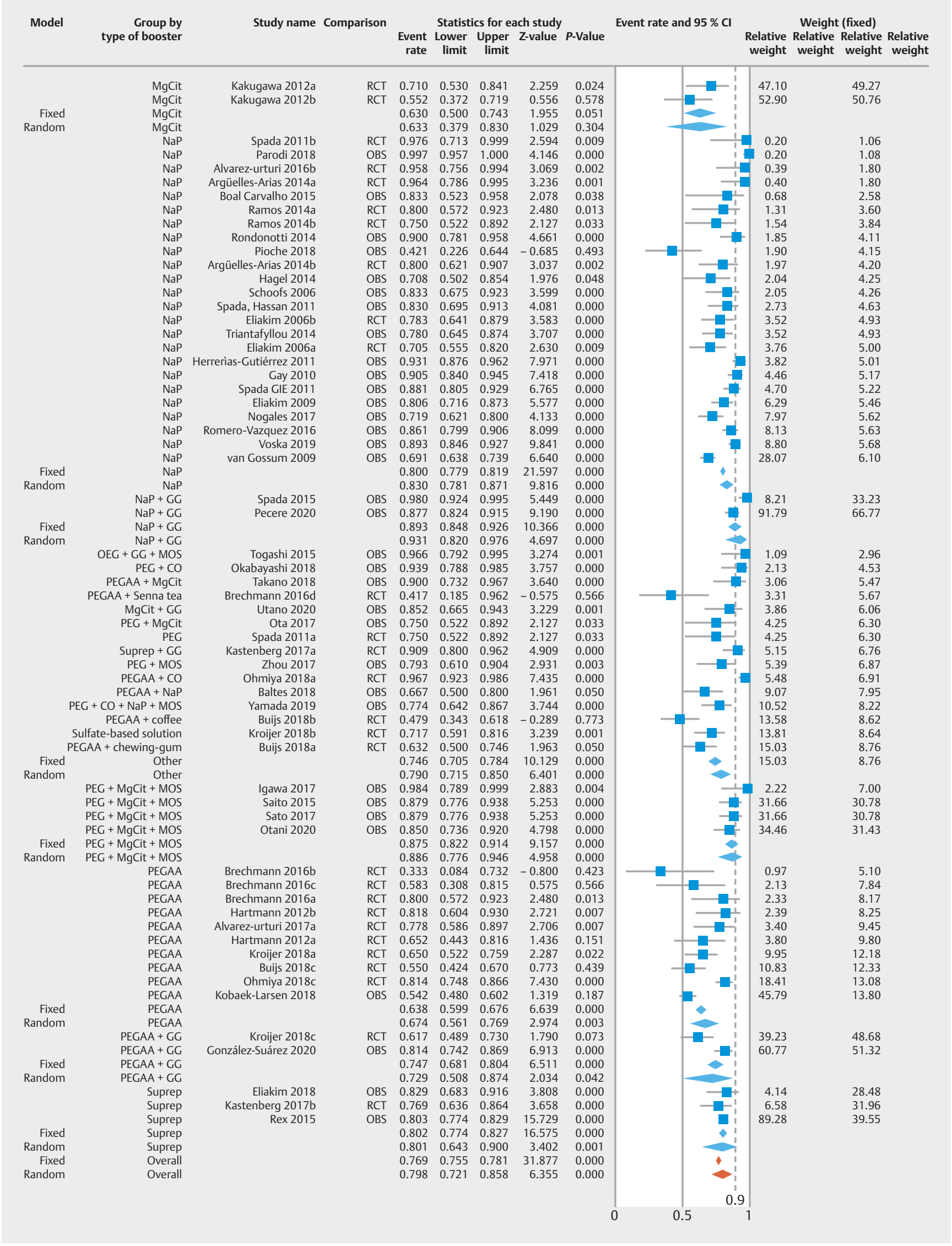

Fig. 2 Forest plot of completeness by booster type. The dashed line represents $90 \%$. CI, confidence interval; CO, castor oil; GG, Gastrografin; MgCit, magnesium citrate; MOS, mosapride; NaP, sodium phosphate; OBS, observational study; PEG, polyethylene glycol; PEGAA, polyethylene glycol and ascorbic acid; RCT, randomized controlled trial. 


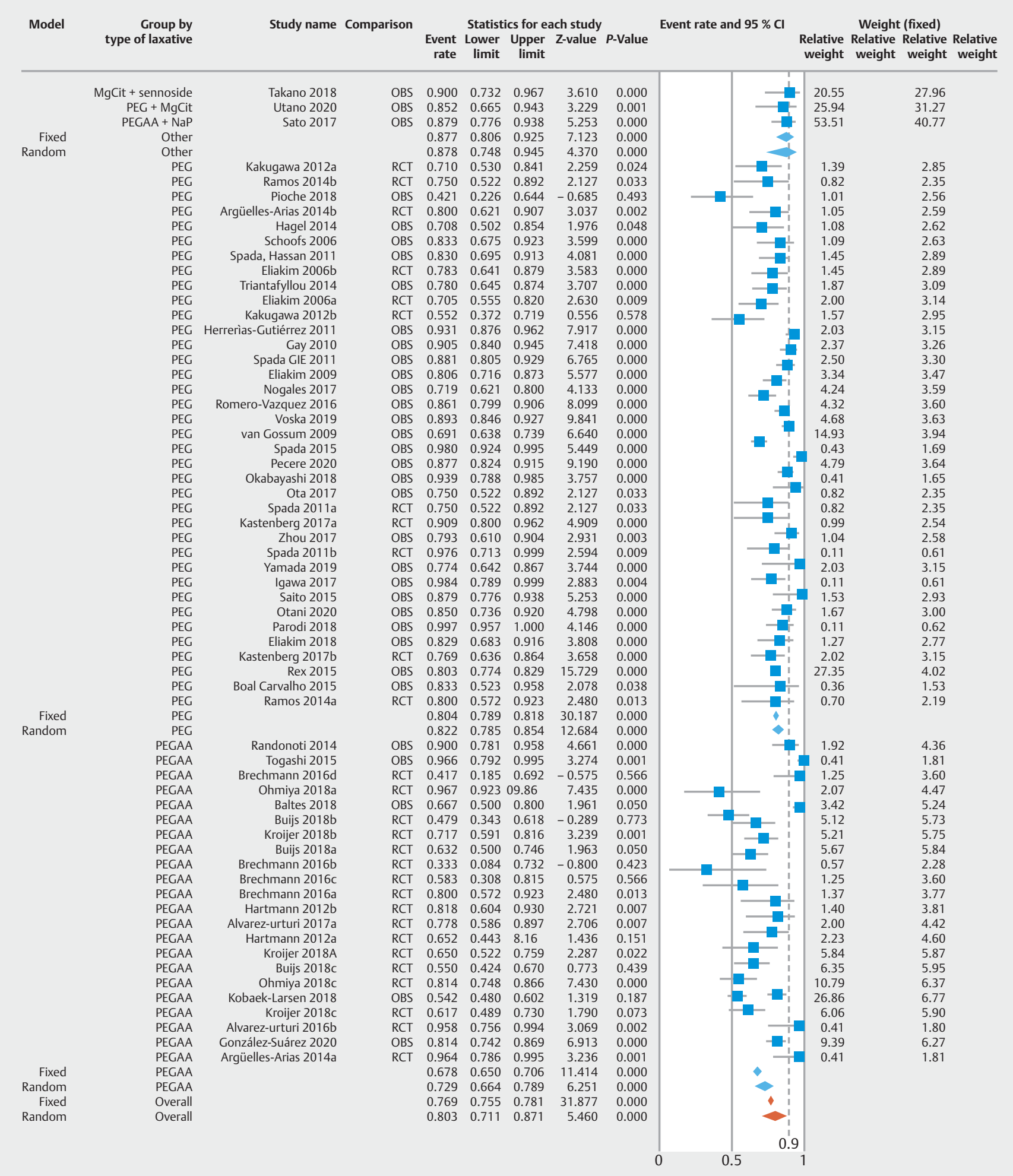

- Fig. 3 Forest plot of completeness by laxative type. The dashed line represents $90 \% . \mathrm{Cl}$, confidence interval; $\mathrm{MgCit}$, magnesium citrate; $\mathrm{NaP}$, sodium phosphate; OBS, observational study; PEG, polyethylene glycol; PEGAA, polyethylene glycol and ascorbic acid; RCT, randomized controlled trial. 


\begin{tabular}{|c|c|c|c|c|c|c|c|c|c|c|c|c|}
\hline \multirow[t]{2}{*}{ Model } & Group by & Study name & Comparison & & Statisti & ics for ea & ach study & & Event rate and $95 \% \mathrm{Cl}$ & & Weight & $t$ (fixed) \\
\hline & prokinetic type & & & $\begin{array}{r}\text { Event } \\
\text { rate }\end{array}$ & $\begin{array}{r}\text { Lower } \\
\text { limit }\end{array}$ & $\begin{array}{r}\text { Upper } \\
\text { limit }\end{array}$ & Z-value & $P$-Value & & $\begin{array}{r}\text { Relative } \\
\text { weight }\end{array}$ & $\begin{array}{r}\text { Relative } \\
\text { weight }\end{array}$ & $\begin{array}{c}\text { Relative Relative } \\
\text { weight weight }\end{array}$ \\
\hline & DOM & Spada 2011b & RCT & 0.976 & 0.713 & 0.999 & 2.594 & 0.009 & & 0.14 & & 0.79 \\
\hline & DOM & Parodi 2018 & OBS & 0.997 & 0.957 & 1.000 & 4.146 & 0.000 & & 0.14 & & 0.81 \\
\hline & DOM & Argüelles-Arias 2014a & RCT & 0.964 & 0.786 & 0.995 & 3.236 & 0.001 & & 0.28 & & 1.35 \\
\hline & DOM & Togashi 2015 & OBS & 0.966 & 0.792 & 0.995 & 3.274 & 0.001 & & 0.28 & & 1.35 \\
\hline & DOM & Boal Carvalho 2015 & OBS & 0.833 & 0.523 & 0.958 & 2.078 & 0.038 & & 0.48 & & 1.94 \\
\hline & DOM & Spada 2015 & OBS & 0.980 & 0.924 & 0.995 & 5.449 & 0.000 & & 0.56 & & 2.13 \\
\hline & DOM & Ramos 2014a & $\mathrm{RCT}$ & 0.800 & 0.572 & 0.923 & 2.480 & 0.013 & & 0.92 & & 2.71 \\
\hline & DOM & Hartmann 2012b & $\mathrm{RCT}$ & 0.818 & 0.604 & 0.930 & 2.721 & 0.007 & & 0.94 & & 2.74 \\
\hline & DOM & Utano 2020 & OBS & 0.852 & 0.665 & 0.943 & 3.229 & 0.001 & & 0.98 & & 2.79 \\
\hline & DOM & Spada 2011a & RCT & 0.750 & 0.522 & 0.892 & 2.127 & 0.033 & & 1.08 & & 2.90 \\
\hline & DOM & Ramos 2014b & RCT & 0.750 & 0.522 & 0.892 & 2.127 & 0.033 & & 1.08 & & 2.90 \\
\hline & DOM & Pioche 2018 & OBS & 0.421 & 0.226 & 0.644 & -0.685 & 0.493 & & 1.33 & & 3.14 \\
\hline & DOM & Argüelles-Arias 2014b & RCT & 0.800 & 0.621 & 0.907 & 3.037 & 0.002 & & 1.38 & & 3.18 \\
\hline & DOM & Schoofs 2006 & OBS & 0.833 & 0.376 & 0.923 & 3.599 & 0.000 & & 1.44 & & 3.22 \\
\hline & DOM & Hartmann 2012a & $\mathrm{RCT}$ & 0.652 & 0.443 & 0.816 & 1.436 & 0.151 & & 1.50 & & 3.27 \\
\hline & DOM & Spada, Hassan 2011 & OBS & 0.830 & 0.695 & 0.913 & 4.081 & 0.000 & & 1.91 & & 3.51 \\
\hline & DOM & Baltes 2018 & OBS & 0.667 & 0.500 & 0.800 & 1.961 & 0.050 & & 2.31 & & 3.68 \\
\hline & DOM & Triantafyllou 2014 & OBS & 0.780 & 0.645 & 0.874 & 3.707 & 0.000 & & 2.47 & & 3.74 \\
\hline & DOM & Herrerìas-Gutiérrez 2011 & OBS & 0.931 & 0.876 & 0.962 & 7.917 & 0.000 & & 2.68 & & 3.81 \\
\hline & DOM & Gay 2010 & OBS & 0.905 & 0.840 & 0.945 & 7.418 & 0.000 & & 3.13 & & 3.93 \\
\hline & DOM & Spada GIE 2011 & OBS & 0.881 & 0.805 & 0.929 & 6.765 & 0.000 & & 3.30 & & 3.96 \\
\hline & DOM & Buijs 2018b & $\mathrm{RCT}$ & 0.479 & 0.343 & 0.618 & -0.289 & 0.773 & i & 3.45 & & 4.00 \\
\hline & DOM & Kroijer 2018b & RCT & 0.717 & 0.591 & 0.816 & 3.239 & 0.001 & & 3.51 & & 4.01 \\
\hline & DOM & Buijs 2018a & RCT & 0.632 & 0.500 & 0.746 & 1.963 & 0.050 & 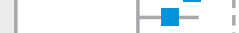 & 3.82 & & 4.06 \\
\hline & DOM & Kroijer 2018a & RCT & 0.650 & 0.522 & 0.759 & 2.287 & 0.022 & 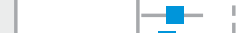 & 3.93 & & 4.08 \\
\hline & DOM & Kroijer 2018c & RCT & 0.617 & 0.489 & 0.730 & 1.790 & 0.073 & I & 4.09 & & 4.11 \\
\hline & DOM & Buijs 2018c & $\mathrm{RCT}$ & 0.550 & 0.424 & 0.670 & 0.773 & 0.439 & 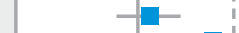 & 4.28 & & 4.13 \\
\hline & DOM & Eliakim 2009 & OBS & 0.806 & 0.716 & 0.873 & 5.577 & 0.000 & & 4.41 & & 4.15 \\
\hline & DOM & Pecere 2020 & OBS & 0.877 & 0.824 & 0.915 & 9.190 & 0.000 & & 6.32 & & 4.33 \\
\hline & DOM & Kobaek-Larsen 2018 & OBS & 0.542 & 0.480 & 0.602 & 1.319 & 0.187 & 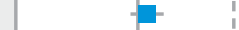 & 18.11 & & 4.64 \\
\hline & DOM & van Gossum 2009 & OBS & 0.691 & 0.638 & 0.739 & 6.640 & 0.000 & i & 19.71 & & 4.66 \\
\hline Fixed & DOM & & & 0.719 & 0.697 & 0.740 & 17.489 & 0.000 & $\bullet$ & & & \\
\hline Random & DOM & & & 0.781 & 0.731 & 0.824 & 9.127 & 0.000 & & & & \\
\hline & ERY & Brechmann 2016b & RCT & 0.333 & 0.084 & 0.732 & -0.800 & 0.423 & & 12.86 & & 17.53 \\
\hline & ERY & Brechmann 2016d & $\mathrm{RCT}$ & 0.417 & 0.185 & 0.692 & -0.575 & 0.566 & H & 28.14 & & 27.11 \\
\hline & ERY & Brechmann 2016c & $\mathrm{RCT}$ & 0.583 & 0.308 & 0.815 & 0.575 & 0.566 & 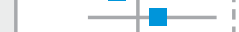 & 28.14 & & 27.11 \\
\hline & ERY & Brechmann 2016a & RCT & 0.800 & 0.572 & 0.923 & 2.480 & 0.013 & & 30.87 & & 28.26 \\
\hline Fixed & ERY & & & 0.584 & 0.433 & 0.721 & 1.091 & 0.275 & & & & \\
\hline Random & ERY & & & 0.567 & 0.352 & 0.760 & 0.602 & 0.547 & & & & \\
\hline & MET & Igawa 2017 & OBS & 0.984 & 0.789 & 0.999 & 2.883 & 0.004 & & 0.23 & & 2.27 \\
\hline & MET & Alvarez-urturi 2016b & RCT & 0.958 & 0.756 & 0.994 & 3.069 & 0.002 & & 0.45 & & 3.83 \\
\hline & MET & Okabayashi 2018 & OBS & 0.939 & 0.788 & 0.985 & 3.757 & 0.000 & & 0.89 & & 5.92 \\
\hline & MET & Rondonotti 2014 & OBS & 0.900 & 0.781 & 0.958 & 4.661 & 0.000 & & 2.12 & & 8.87 \\
\hline & MET & Alvarez-urturi 2017a & RCT & 0.778 & 0.586 & 0.897 & 2.706 & 0.007 & & 2.20 & & 8.98 \\
\hline & MET & Eliakim 2018 & OBS & 0.829 & 0.683 & 0.916 & 3.808 & 0.000 & & 2.74 & & 9.64 \\
\hline & MET & Sato 2017 & OBS & 0.879 & 0.776 & 0.938 & 5.253 & 0.000 & & 3.32 & & 10.17 \\
\hline & MET & Nogales 2017 & OBS & 0.719 & 0.621 & 0.800 & 4.133 & 0.000 & & 9.16 & & 12.21 \\
\hline & MET & Romero-Vazquez 2016 & OBS & 0.861 & 0.799 & 0.906 & 8.099 & 0.000 & & 9.35 & & 12.23 \\
\hline & MET & González-Suárez 2020 & OBS & 0.814 & 0.742 & 0.869 & 6.913 & 0.000 & - & 10.38 & & 12.37 \\
\hline & MET & Rex 2015 & OBS & 0.803 & 0.774 & 0.829 & 15.729 & 0.000 & - & 59.15 & & 13.50 \\
\hline Fixed & MET & & & 0.813 & 0.792 & 0.833 & 21.420 & 0.000 & $\rightarrow$ & & & \\
\hline Random & MET & & & 0.852 & 0.783 & 0.901 & 7.425 & 0.000 & 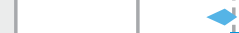 & & & \\
\hline & MET or ERY & Kastenberg 2017a & RCT & 0.909 & 0.800 & 0.962 & 4.909 & 0.000 & & 32.99 & & 45.07 \\
\hline & MET or ERY & Kastenberg 2017b & $\mathrm{RCT}$ & 0.769 & 0.636 & 0.864 & 3.658 & 0.000 & & 67.01 & & 54.93 \\
\hline Fixed & MET or ERY & & & 0.827 & 0.739 & 0.890 & 5.814 & 0.000 & & & & \\
\hline Random & MET or ERY & & & 0.845 & 0.660 & 0.969 & 3.210 & 0.001 & & & & \\
\hline & MOS & Takano 2018 & OBS & 0.900 & 0.732 & 0.967 & 3.610 & 0.000 & & 6.51 & & 17.99 \\
\hline & MOS & Kakugawa 2012a & RCT & 0.710 & 0.530 & 0.841 & 2.259 & 0.024 & & 15.39 & & 24.88 \\
\hline & MOS & Kakugawa 2012b & RCT & 0.552 & 0.372 & 0.719 & 0.556 & 0.578 & & 17.28 & & 25.67 \\
\hline & MOS & Ohmiya 2018 c & RCT & 0.814 & 0.748 & 0.866 & 7.430 & 0.000 & & 60.83 & & 31.47 \\
\hline Fixed & MOS & & & 0.771 & 0.713 & 0.821 & 7.832 & 0.000 & & & & \\
\hline Random & MOS & & & 0.757 & 0.600 & 0.866 & 3.049 & 0.002 & & & & \\
\hline & NA & Ota 2017 & OBS & 0.750 & 0.522 & 0.892 & 2.127 & 0.033 & & 12.34 & & 17.34 \\
\hline & NA & Zhou 2017 & OBS & 0.793 & 0.610 & 0.904 & 2.931 & 0.003 & & 15.65 & & 18.94 \\
\hline & NA & Hagel 204 & OBS & 0.708 & 0.502 & 0.854 & 1.976 & 0.048 & a & 16.31 & & 19.21 \\
\hline & NA & Otani 2020 & OBS & 0.850 & 0.736 & 0.920 & 4.798 & 0.000 & -1 & 25.16 & & 21.77 \\
\hline & NA & Yamada 2019 & OBS & 0.774 & 0.642 & 0.867 & 3.744 & 0.000 & -7 & 30.54 & & 22.75 \\
\hline Fixed & NA & & & 0.786 & 0.720 & 0.840 & 7.180 & 0.000 & 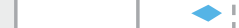 & & & \\
\hline Random & NA & & & 0.781 & 0.647 & 0.874 & 3.738 & 0.000 & & & & \\
\hline & $\mathrm{MET} / \mathrm{MgCit}+\mathrm{MOS}$ & Ohmiya 2018a & RCT & 0.967 & 0.923 & 0.986 & 7.435 & 0.000 & & 14.52 & & 28.77 \\
\hline & MET, MOS & Saito 2015 & OBS & 0.879 & 0.776 & 0.938 & 5.253 & 0.000 & & 21.11 & & 32.18 \\
\hline & MET & Voska 2019 & OBS & 0.893 & 0.846 & 0.927 & 9.841 & 0.000 & & 64.37 & & 39.05 \\
\hline Fixed & Other & & & 0.907 & 0.874 & 0.932 & 13.142 & 0.000 & & 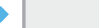 & & \\
\hline Random & Other & & & 0.920 & 0.835 & 0.963 & 5.832 & 0.000 & & & & \\
\hline & Tegaserod & Eliakim 2006b & $\mathrm{RCT}$ & 0.783 & 0.641 & 0.879 & 3.583 & 0.000 & -1 & 46.08 & & 49.11 \\
\hline & Tegaserod & Eliakim 2006a & $\mathrm{RCT}$ & 0.705 & 0.555 & 0.820 & 2.630 & 0.009 & $\rightarrow-$ - & 53.92 & & 50.89 \\
\hline Fixed & Tegaserod & & & 0.742 & 0.642 & 0.823 & 4.364 & 0.000 & $<1$ & & & \\
\hline Random & Tegaserod & & & 0.745 & 0.518 & 0.888 & 2.101 & 0.036 & -1 & & & \\
\hline Fixed & Overall & & & 0.769 & 0.755 & 0.781 & 31.877 & 0.000 & $\rightarrow i$ & & & \\
\hline Random & Overall & & & 0.798 & 0.724 & 0.857 & 6.563 & 0.000 & $<$ & & & \\
\hline & & & & & & & & & 0. & & & \\
\hline & & & & & & & & & 0.5 & 1 & & \\
\hline
\end{tabular}

Pig. 4 Forest plot by completeness by prokinetic type. The dashed line represents $90 \%$. Cl, confidence interval; DOM, domperidone; ERY, erythromycin; MET, metoclopramide; MgCit, magnesium citrate; MOS, mosapride; NA, not available; OBS, observational study; RCT, randomized controlled trial 
$0.764,95 \% \mathrm{Cl}, 0.612-0.869)$. Considerable heterogeneity was found between studies $\left(I^{2}=96.25, P=0.000\right)$.

The subgroup analyses revealed that CR differed regarding laxative type, type of laxative administration, booster type, prokinetics use, and prokinetics types with the highest observed $C R$ for $\mathrm{NaP}+\mathrm{GG}$ booster $(n=2, E R=0.931,95 \% \mathrm{Cl}$ : 0.820 to $0.976, Z=4.697 P=0.000)$, NaP-based booster $(n=$ 28, $\mathrm{ER}=0.834,95 \% \mathrm{Cl}: 0.788$ to $0.871, \mathrm{Z}=10.640 P=0.000)$, split regimen of laxative $(n=50, E R=0.799,95 \% \mathrm{Cl}: 0.762$ to $0.863)$ and type of prokinetics ( $n=3, E R=0.920,95 \% \mathrm{Cl}: 0.835$ to $0.888, Z=2.101 P=0.000)$. Except for split vs. non-split regimen of laxative administration and usage of prokinetics (Yes vs. No) there were significant heterogeneities between studies regarding all effect size moderators. The highest $Q$ value was calculated for booster types $(Q=19.581, d f=7, P=0.007)$ while the lowest for booster type grouped on NaP presence $(Q=$ 4.523, $\mathrm{df}=1, P=0.033$ ).

A meta-regression using a random-effects model revealed that the model utilizing different CR moderators (laxative total volume without booster, \% of males, mean age, number of study participants, type of laxative with particular administration regimen (Split vs. non-spilt), type of booster, and usage and type of prokinetics), explained a total of $25 \%$ of variance within the event rates. A meta-regression using a random-effects model revealed that the model utilizing CCE device (CCE1 as reference group) had no impact on any of the studied outcomes (Cleanliness: $Q=2.5, \mathrm{df}=3, P=0.475$; Completeness: $\mathrm{Q}$ =1.98, $\mathrm{df}=3, P=0.5764$; Transit time: $Q=1.26, \mathrm{df}=1, P=$ $0.2624)$. Furthermore, the robustness of results we obtained were subjected to a sensitivity analysis (without subgroup analyses). The exclusion of one study at a time showed the results are not influenced by the effect size of only one study. The effect sizes ranged between 0.763 and 0.772 (random 0.768 ; lower limit: $0.734-0.741$ and upper limit: $0.798-0.801 ; P=$ 0.000 ), 0.792 and 0.802 (random 0.798; lower limit: 0.758 0.802 and upper limit: $0.823-0.832 ; P=0.000), 1.842$ and 2.034 (random 1.972; standard error between 0.13-0.166, lower limit: 1.588-1.709 and upper limit: 2.097-2.36; $P=$ $0.000)$ for cleanliness, completeness and transit time respectively.

Overall, an Egger's test suggested a publication bias regarding the event rate (Egger's test: $P=0.0276$ (Supplementary Fig. 1). The Duval and Tweedie method adjusted values of 13 studies to left of mean; random model point estimate: 0.753 ; $95 \% \mathrm{Cl}: 0.712-0.789, \mathrm{Q}$ value $=484.39$.

\section{Regimen effects on colon cleanliness}

Using random-effects weights, the overall rate of ACR was 0.768 (95\% Cl, 0.735-0.797) ( Fig.5). Between-study heterogeneity was present for ACR by booster, laxative, and prokinetic type $\left(I^{2}=96.25, P=0.000\right)$. Depending on the type of laxatives, ACR for PEG was $0.790(n=37,95 \% \mathrm{Cl}, 0.750-0.826)$; for PEGAA $0.716(n=22,95 \% \mathrm{Cl}, 0.663-0.7729)$. We did not find any effect of split-regimen compared to non-split regimen on ACR with rates with $0.766(95 \% \mathrm{Cl}, 0.730-0.798)$ and 0.781 (95\% Cl, 0.690-0.850), respectively. Among boosters, PEG + $\mathrm{MgCit}(n=4)$ had the highest rate of $A C R=0.953(95 \% \mathrm{Cl}$,
0.896-0.979) ( Fig. 6). The overall ACR for studies using prokinetics was $0.778(95 \% \mathrm{Cl}, 0.697-0.843)$ (॰ Fig. 7) (Supplementary Fig. 2).

A meta-regression using a random-effects model revealed that the model utilizing the abovementioned moderators explained a total of $30 \%$ of variance within the event rates for cleanliness $(\mathrm{Q}=62.4, \mathrm{df}=23, P=0.0000)$. Overall, an Egger's test did not suggest a publication bias regarding the ACR (Egger's test: $P=0.766$ ) (Supplementary Fig. 3).

\section{Regimen effects on colonic transit time}

Using random-effects weights, the overall mean CTT was 197.2 minutes (95\% Cl, 166.7-227.9). Between-study heterogeneity was present for CTT by booster and laxative type $\left(I^{2}=96.25, P\right.$ $=0.000)$. The subgroup analyses revealed that mean CTT differed regarding booster ( $>$ Fig.8, Supplementary Fig. 2) and laxative types ( $>$ Fig. 9, Supplementary Fig. 2), with the lowest mean observed for PEG as laxative $(N=10$, mean $=178.6,95 \%$ Cl, 191-291.7).

A meta-regression using a random-effects model with all study moderators was not possible due to problems with collinearity. Thus, all effect size moderators were separately analyzed. We were able to show that the variance in mean CTT was explained in $40 \%$ by booster type $(Q=8.95, \mathrm{df}=2, P=$ $0.0114)$ ), in $34 \%$ by laxative type $(Q=4.38, d f=1, P=0.0363)$, in $44 \%$ by booster volume $(\mathrm{Q}=14.32, \mathrm{df}=1, P=0.0002)$, and in $43 \%$ with laxative volume with booster $(\mathrm{Q}=8.48, \mathrm{df}=1, P=$ $0.0036)$.

Overall, an Egger's test suggested a publication bias regarding the CTT (Egger's test: $P=0.00727$ ) ( $>$ Fig. 7-9). The Duval and Tweedie method adjusted values of 5 studies to left of mean; random model point estimate: 154.24 ; $95 \% \mathrm{Cl}$ : $118.81-$ 189.66, $Q$ value $=197.86$.

\section{Discussion}

This is, to date, the most extensive systematic review and metaanalysis of studies that report colon cleanliness and completeness in CCE. Interestingly, by pooling all the included studies, both CCE CRs and colon cleansing are suboptimal. NaP was the most frequently used booster with a completion rate of 0.830 ( $95 \% \mathrm{Cl}, 0.781-0.871)$. We found that the highest rate of CCE completion was achieved in the two studies where $\mathrm{NaP}+\mathrm{GG}$ booster was used with a CR of 0.931 ( $95 \% \mathrm{Cl}, 0.820$ to 0.976 ). Regarding colon cleanliness, a single study reported adequate colonic cleanliness of 0.939 (95\% Cl, 0.849-0.977) using PEG + MgCit. PEG only was used in most studies with a reported pooled rate of adequate cleanliness of 0.790 (95\% Cl, $0.750-$ 0.826 ). Boosters are, of course, required in CCE to increase the capsule completion rate and ensure complete visualization of the colonic and rectal mucosa.

The European Society of Gastrointestinal Endoscopy (ESGE) recommends NaP-based boosters for CCE [57]. However, their usage is restricted by possible toxicity in patients with renal and cardiovascular disease, where serious electrolyte imbalance has been reported [58]. The recent ESGE-guidelines for flexible colonoscopy recommends against the routine use of 


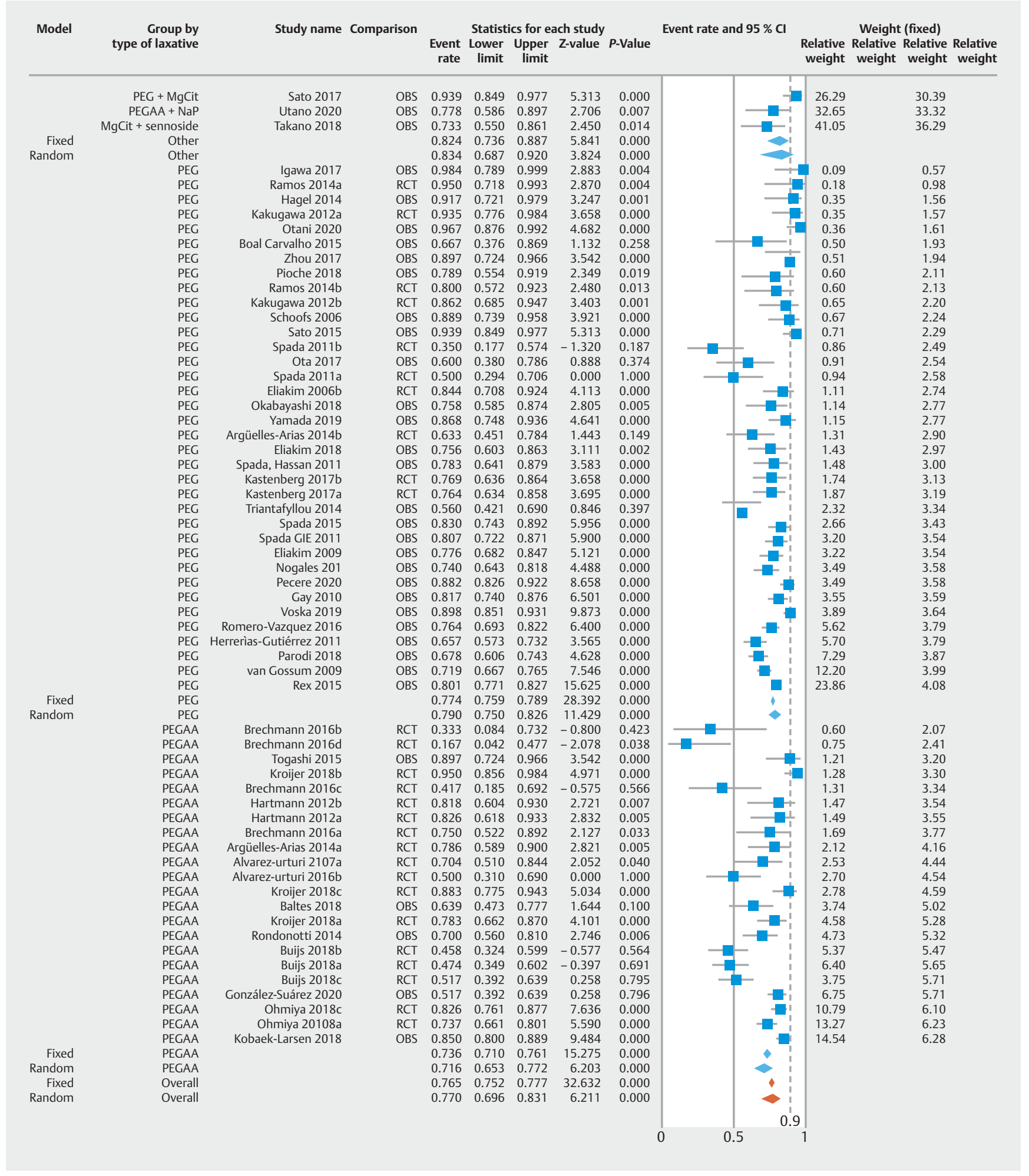

- Fig. 5 Forest plot of cleanliness by laxative type. The dashed line represents $90 \%$. Cl, confidence interval; MgCit, magnesium citrate; NaP, sodium phosphate; OBS, observational study; PEG, polyethylene glycol; PEGAA, polyethylene glycol and ascorbic acid; RCT, randomized controlled trial.

oral NaP for bowel preparation [59]. Moreover, oral NaP solution has been withdrawn from the market in some countries [60]. The decreased use of $\mathrm{NaP}$ and attempt to replace it with a new effective and safe booster is obvious in our review, show-

ing that during the last 4 years, only seven of 29 (24\%) compar- 


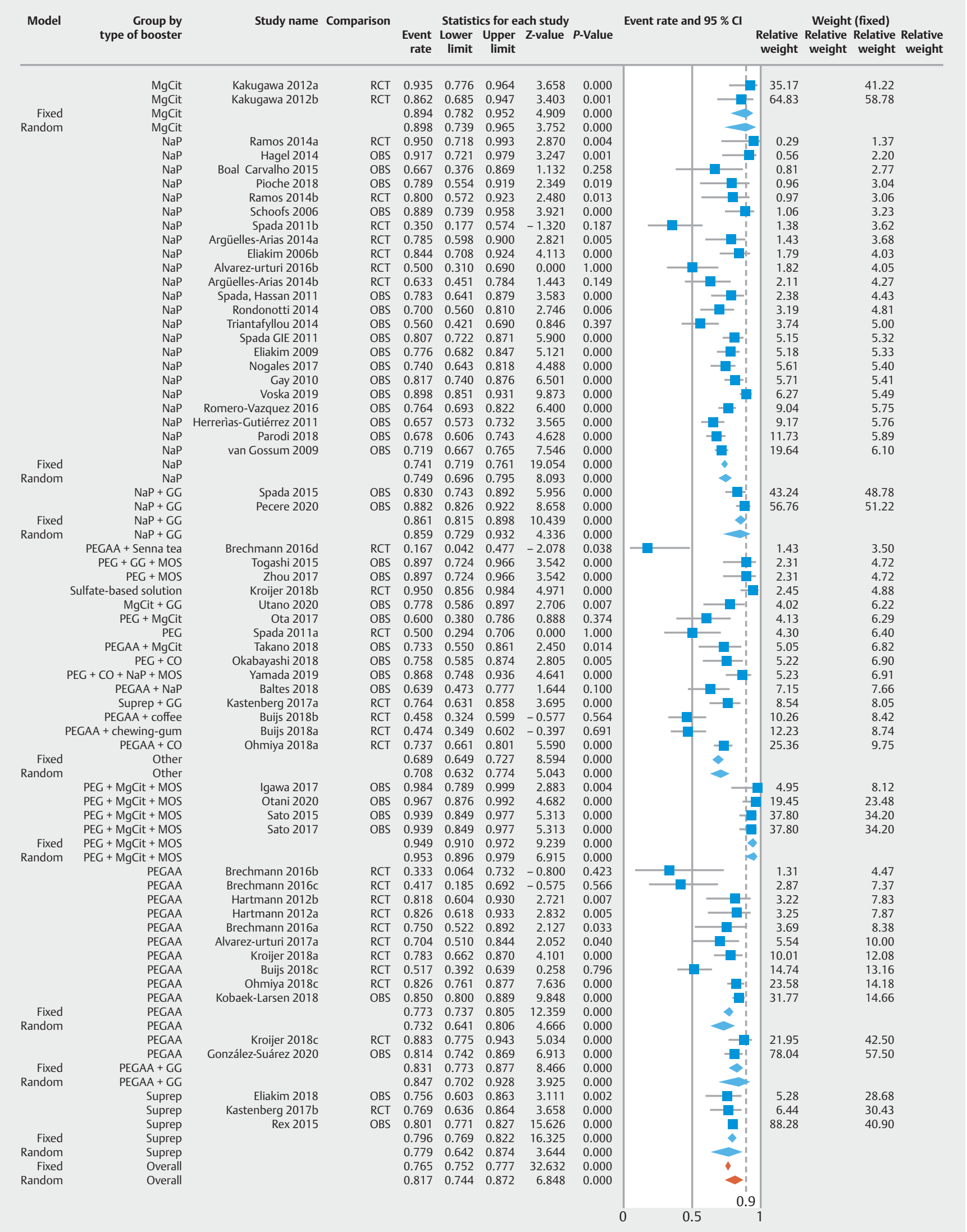

Fig. 6 Forest plot of cleanliness by booster type. The dashed line represents $90 \%$.Cl, confidence interval; CO, castor oil; GG, Gastrografin; MgCit, magnesium citrate; MOS, mosapride; NaP, sodium phosphate; OBS, observational study; PEG, polyethylene glycol; PEGAA, polyethylene glycol and ascorbic acid; RCT, randomized controlled trial. 


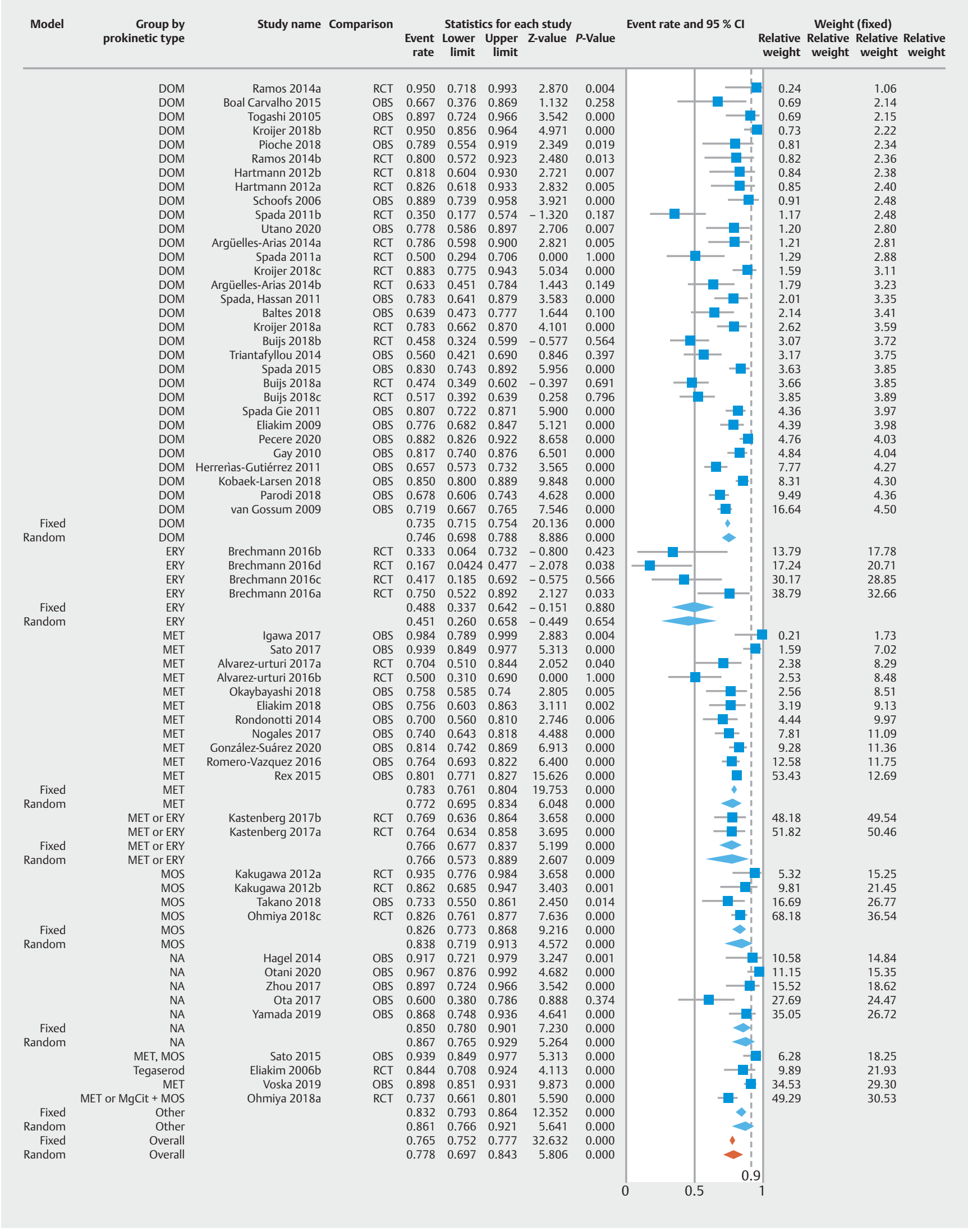

Fig. 7 Forest plot of cleanliness by prokinetic type. The dashed line represents $90 \%$. CI, confidence interval; DOM, domperidone; ERY, erythromycin; MET, metoclopramide; MgCit, magnesium citrate; MOS, mosapride; NA, not available; OBS, observational study; RCT, randomized controlled trial. 


\begin{tabular}{|c|c|c|c|c|c|c|c|c|c|c|c|c|c|c|c|}
\hline \multirow[t]{11}{*}{ Model } & \multirow{3}{*}{$\begin{array}{r}\text { Group by } \\
\text { booster type }\end{array}$} & \multirow{3}{*}{$\begin{array}{l}\text { Study name } \\
\text { Hagel } 2014\end{array}$} & \multirow{3}{*}{$\begin{array}{r}\text { Comparison } \\
\text { RCT }\end{array}$} & \multirow{3}{*}{$\begin{array}{l}\text { Mean } \\
3.090\end{array}$} & \multirow{3}{*}{$\begin{array}{r}\begin{array}{r}\text { Standard } \\
\text { error }\end{array} \\
0.414\end{array}$} & \multicolumn{3}{|c|}{ Statistics for each study } & \multirow[b]{2}{*}{ Z-value } & \multirow[b]{2}{*}{$P$-Value } & \multirow{2}{*}{\multicolumn{2}{|c|}{$\begin{array}{l}\text { Mean and } \\
95 \% \mathrm{Cl}\end{array}$}} & \multirow{2}{*}{$\begin{array}{r}\text { Relative } \\
\text { weight }\end{array}$} & \multirow{2}{*}{$\begin{array}{l}\text { Weight } \\
\text { Relative } \\
\text { weight }\end{array}$} & \multirow{2}{*}{$\begin{array}{l}\text { (fixed) } \\
\text { Relative Relative } \\
\text { weight weight }\end{array}$} \\
\hline & & & & & & Variance & $\begin{array}{r}\text { Lower } \\
\text { limit }\end{array}$ & $\begin{array}{c}\text { Upper } \\
\text { limit }\end{array}$ & & & & & & & \\
\hline & & & & & & 0.172 & 2.278 & 3.902 & 7.457 & 0.000 & & - & 3.73 & & 7.73 \\
\hline & $\mathrm{NaP}$ & Argüelles-Arias 2014a & RCT & 1.600 & 0.350 & 0.122 & 0.915 & 2.285 & 4.576 & 0.000 & $\rightarrow$ & & 5.24 & & 9.07 \\
\hline & $\mathrm{NaP}$ & Ramos 2014b & RCT & 1.670 & 0.326 & 0.107 & 1.030 & 2.310 & 5.115 & 0.000 & $\rightarrow$ & & 6.01 & & 9.59 \\
\hline & $\mathrm{NaP}$ & Ramos 2014a & RCT & 1.620 & 0.257 & 0.066 & 1.116 & 2.124 & 6.300 & 0.000 & $\rightarrow$ & & 9.68 & & 11.27 \\
\hline & $\mathrm{NaP}$ & Rondonotti 2014 & OBS & 2.130 & 0.245 & 0.060 & 1.650 & 2.610 & 8.706 & 0.000 & & & 10.70 & & 11.59 \\
\hline & $\mathrm{NaP}$ & Spada 2011b & RCT & 1.370 & 0.230 & 0.053 & 0.919 & 1.821 & 5.948 & 0.000 & - & & 12.07 & & 11.95 \\
\hline & $\mathrm{NaP}$ & Eliakim 2006b & RCT & 1.700 & 0.195 & 0.038 & 1.319 & 2.081 & 8.735 & 0.000 & - & & 16.91 & & 12.85 \\
\hline & $\mathrm{NaP}$ & Eliakim 2006a & RCT & 1.770 & 0.193 & 0.037 & 1.392 & 2.148 & 9.173 & 0.000 & $\rightarrow$ & & 17.20 & & 12.89 \\
\hline & $\mathrm{NaP}$ & Argüelles-Arias 2014b & RCT & 1.460 & 0.186 & 0.035 & 1.095 & 1.825 & 7.840 & 0.000 & $=$ & & 18.47 & & 13.05 \\
\hline Fixed & $\mathrm{NaP}$ & & & 1.711 & 0.080 & 0.006 & 1.554 & 1.868 & 21.381 & 0.000 & $\leftrightarrow$ & & & & \\
\hline \multirow[t]{4}{*}{ Random } & $\mathrm{NaP}$ & & & 1.775 & 0.161 & 0.026 & 1.459 & 2.091 & 11.005 & 0.000 & $\gamma$ & & & & \\
\hline & PEG & Spada 2011a & RCT & 3.320 & 0.387 & 0.150 & 2.562 & 4.078 & 8.582 & 0.000 & & $\rightarrow$ & $\rightarrow \quad 5.12$ & & 22.33 \\
\hline & Suprep & Kastenberg 2017a & RCT & 1.320 & 0.129 & 0.017 & 1.066 & 1.574 & 10.197 & 0.000 & $=$ & & 45.71 & & 38.71 \\
\hline & Suprep + GG & Kastenberg 2017b & RCT & 1.560 & 0.125 & 0.016 & 1.315 & 1.805 & 12.499 & 0.000 & 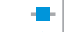 & & 49.17 & & 38.96 \\
\hline Fixed & Other & & & 1.540 & 0.088 & 0.008 & 1.369 & 1.712 & 17.601 & 0.000 & $\bullet$ & & & & \\
\hline \multirow[t]{3}{*}{ Random } & Other & & & 1.860 & 0.265 & 0.070 & 1.341 & 2.379 & 7.022 & 0.000 & & & & & \\
\hline & PEGAA & Hartmann 2012a & RCT & 2.480 & 0.296 & 0.088 & 1.900 & 3.060 & 8.376 & 0.000 & & - & 46.32 & & 48.78 \\
\hline & PEGAA & Hartmann 2012b & RCT & 3.340 & 0.275 & 0.076 & 2.801 & 3.879 & 12.144 & 0.000 & & - & 53.68 & & 51.22 \\
\hline Fixed & PEGAA & & & 2.942 & 0.202 & 0.041 & 2.547 & 3.337 & 14.598 & 0.000 & & $<$ & & & \\
\hline Random & PEGAA & & & 2.921 & 0.351 & 0.123 & 2.233 & 3.608 & 8.327 & 0.000 & & $\Longrightarrow$ & & & \\
\hline Fixed & Overall & & & 1.737 & 0.057 & 0.003 & 1.626 & 1.848 & 30.645 & 0.000 & $\bullet$ & & & & \\
\hline \multirow[t]{2}{*}{ Random } & Overall & & & 2.128 & 0.325 & 0.105 & 1.492 & 2.765 & 6.559 & 0.000 & & & & & \\
\hline & & & & & & & & & & & & & 4.00 & & \\
\hline
\end{tabular}

- Fig. 8 Forest plot of transit time by booster type. Cl, confidence interval; GG, Gastrografin, NaP, sodium phosphate; OBS, observational study; PEGAA, polyethylene glycol and ascorbic acid; RCT, randomized controlled trial.

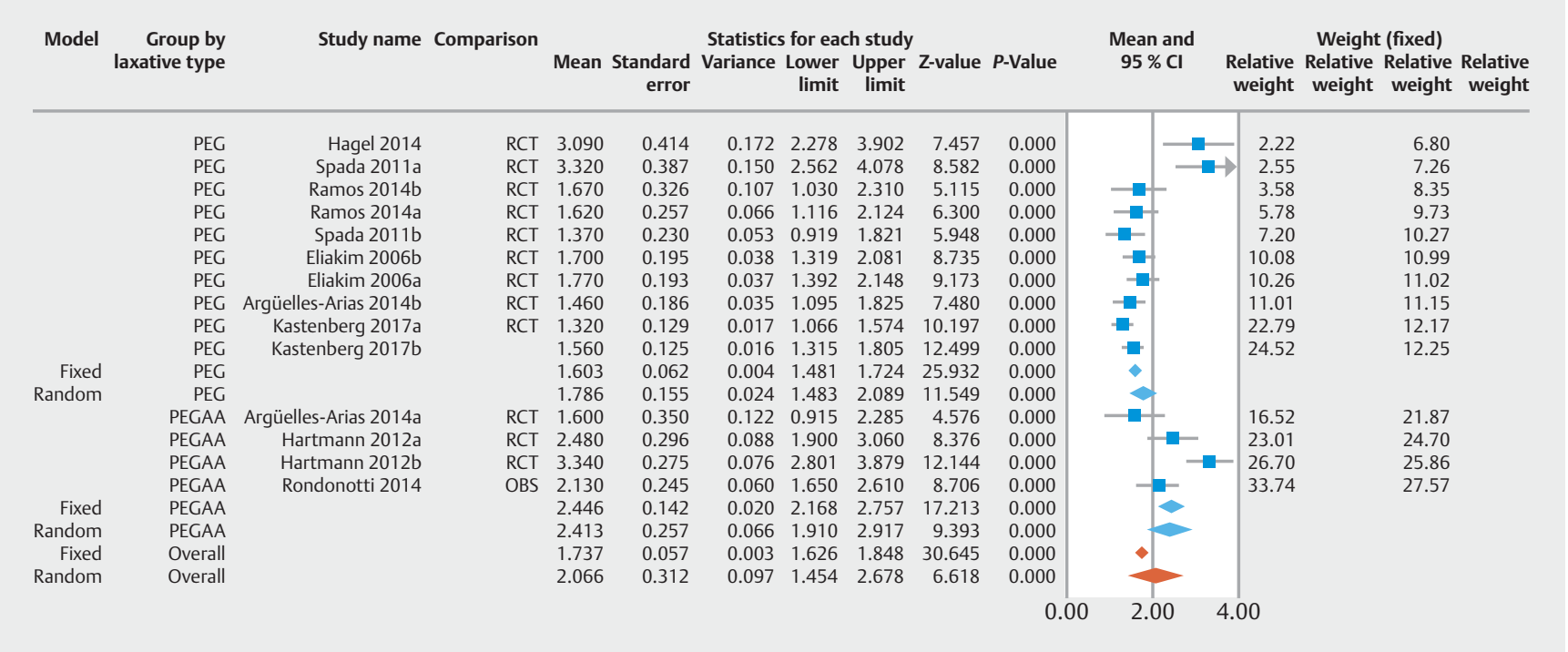

- Fig. 9 Forest plot of transit time by laxative type. Cl, confidence interval; OBS, observational study; PEG, polyethylene glycol; PEGAA, polyethylene glycol and ascorbic acid; RCT, randomized controlled trial.

ed to 21 of 35 (60\%) in the previous years published studies included NaP booster in the CCE preparation regimen.

Recent research on CCE highlighted its diagnostic potential in different populations. Many countries have introduced a CRC screening program, which substantially increases the number of performed colonoscopies [61]. This high demand for colonoscopies raises concerns about the long-term sustainability of healthcare systems, especially in times of economic hardship or other occasions such as pandemics $[62,63]$. A possible diagnostic alternative to relieve this burden is CCE. The technology of the CEE design is evolving and algorithms for polyp detection and quality of cleanliness is being developed [64]. Recent work has shown promising results on a polyp matching algorithm with high accuracy compared to the reading of CCE by human readers [65]. Because CCE is only a diagnostic tool without the capabilities for biopsy or polyp removal, populations with a low prevalence of colorectal lesions, and therefore, low need for a subsequent colonoscopy are ideal for CCE. The introduction of CCE as a filter examination in screening programs comes with the caveat that a high completion rate and adequate bowel cleanliness are prerequisite $[66,67]$. In a recent study by Cash et al. comparing the DY of CCE versus computed tomography colography (CTC) in a screening population, CCE was superior to CTC for detection of polyps $\geq 6 \mathrm{~mm}$ but inferior to colonosco- 
py [68]. The CR and ACR of CCE should reach at least $90 \%$ according to quality parameters for flexible colonoscopy defined by international guidelines $[69,70]$.

The importance of adequate bowel cleanliness is emphasized because cancer and polyp miss rate are increased with inadequate visualization of the colonic mucosa [71]. A study of 253 participants from a screening population demonstrated high accuracy of CCE in detecting clinically significant polyps, but only $54 \%$ had adequate visualization of the colon [34]. Taking this into account, together with findings in this detailed review, completion and adequate colonic cleanliness rates need to be improved to meet the ESGE standards for colonoscopy and be a reliable alternative to CC. Currently, a large Danish RCT in a bowel cancer screening population is underway and the study is expected to be completed at the beginning of 2022 [72].

A previous systematic review from 2014, including 12 studies and 1,249 patients, found that PEG and NaP were the most used laxative and booster, respectively [73]. Our meta-analysis supports these findings; despite including 46 studies and a total of 5,003 patients, we were not able to show that PEG or NaP was superior to other used laxatives or boosters at a level of statistical significance. A major strenght of our meta-analysis was a larger number of studies and > 5,000 patients who underwent CCE. Furthermore, most studies were of high quality with prospective designs. Certain limitations of this review should be addressed. Between-study heterogeneity was present for $C R$, $A C R$, and CTT indicated by high $\mathrm{I}^{2}$ values, which need to be considered when interpreting the results. More well-designed protocolized studies would have enabled us to be more conclusive regarding the most optimal bowel preparation regimen. From a clinical standpoint, the quality of colon cleansing is dependent upon several factors, e. g., diet restriction, laxative type, laxative volume, laxative timing of administration, type of boosters, volume of boosters, number of boosters, timing of boosters etc, whereas, in our study we evaluate the impact of each major factor separately. Furthermore, we did not address the patient tolerability, which is a key factor to evaluate the applicability of a preparation regimen in clinical practice.

In terms of future research, we stress the need for large prospective studies on different bowel preparation regimens as much work remains to improve rates of completeness and adequate bowel cleanliness to qualify CCE as an equivalent to CC in certain settings. In parallel, patient tolerability of preparation regimens must be adressed in to avoid dropouts. In times of pandemics such as Covid-19, CCE might offer a important advantage compared to colonoscopy as it can be perfomed in out-patient clinics with minimal contact to health care personal and patients [74].

\section{Conclusions}

In the largest sysyematic review and meta-analysis on CCE, we found that both CRs and ACRs are suboptimal. PEG laxative and $\mathrm{NaP}$ booster were the most commenly used. We were not able to show any superiority concerning completeness or cleanliness rate for any of the abundant laxatives or boosters at a level of statistical significance. Well-designed studies focusing on the completeness or cleanliness of CCE should be performed to identify the optimal preparation regimen.

\section{Competing interests}

Dr. Koulaouzidis is consultant for Jinshan. He is director of iCERV Ltd and cofounder of AJM Medicaps Ltd. He has received a Given Imaging Ltd-ESGE grant, and material support for clinical research from SynMed/Intromedic. In the last 10 years, he has received honoraria and lecture fees from Jinshan, Dr Falk Pharma UK, and Ferring. He has also received educational travel support from Aquilant, Jinshan, Dr Falk Pharma, Almirall, Ferring, and has participated in advisory board meetings for Tillots, Ankon, and Dr Falk PharmaUK. Dr. Toth has received research grant from the Swedish Cancer Society and the Swedish ALF-agreement.

\section{References}

[1] Fireman Z, Kopelman Y. The colon - the latest terrain for capsule endoscopy. Dig Liver Dis 2007; 39: 895-899

[2] Wang YC, Pan J, Liu YW et al. Adverse events of video capsule endoscopy over the past two decades: a systematic review and proportion meta-analysis. BMC Gastroenterol 2020; 20: 364

[3] Kjolhede T, Olholm AM, Kaalby L et al. Diagnostic accuracy of capsule endoscopy compared with colonoscopy for polyp detection: systematic review and meta-analyses. Endoscopy 2020: doi:10.1055/a1249-3938

[4] Sieg A. Capsule endoscopy compared with conventional colonoscopy for detection of colorectal neoplasms. World J Gastrointest Endosc 2011; 3: 81-85

[5] Leighton JA, Rex DK. A grading scale to evaluate colon cleansing for the PillCam COLON capsule: a reliability study. Endoscopy 2011; 43: 123-127

[6] Moher D, Liberati A, Tetzlaff J et al. Preferred reporting items for systematic reviews and meta-analyses: the PRISMA statement. J Clin Epidemiol 2009; 62: 1006-1012

[7] von Elm E, Altman DG, Egger M et al. The Strengthening the Reporting of Observational Studies in Epidemiology (STROBE) statement: guidelines for reporting observational studies. Lancet 2007; 370: 1453-1457

[8] Higgins JP, Altman DG, Gotzsche PC et al. The Cochrane Collaboration's tool for assessing risk of bias in randomised trials. BMJ 2011; 343: d5928 doi: $10.1136 /$ bmj.d5928

[9] vEgger M, Davey Smith G, Schneider M et al. Bias in meta-analysis detected by a simple, graphical test. BMJ 1997; 315: 629-634

[10] Duval S, Tweedie R. Trim and fill: A simple funnel-plot-based method of testing and adjusting for publication bias in meta-analysis. Biometrics 2000; 56: 455-463

[11] Schoofs N, Deviere J, Van Gossum A. PillCam colon capsule endoscopy compared with colonoscopy for colorectal tumor diagnosis: a prospective pilot study. Endoscopy 2006; 38: 971-977

[12] Van Gossum A, Munoz-Navas M, Fernandez-Urien I et al. Capsule endoscopy versus colonoscopy for the detection of polyps and cancer. N Engl J Med 2009; 361: 264-270

[13] Eliakim R, Yassin K, Niv Y et al. Prospective multicenter performance evaluation of the second-generation colon capsule compared with colonoscopy. Endoscopy 2009; 41: 1026-1031

[14] Gay G, Delvaux M, Frederic M et al. Could the colonic capsule PillCam Colon be clinically useful for selecting patients who deserve a com- 
plete colonoscopy? results of clinical comparison with colonoscopy in the perspective of colorectal cancer screening Am J Gastroenterol 2010; 105: 1076-1086

[15] Spada C, Hassan C, Munoz-Navas M et al. Second-generation colon capsule endoscopy compared with colonoscopy. Gastrointest Endosc 2011; 74: 581-589

[16] Herrerias-Gutierrez JM, Arguelles-Arias F, Caunedo-Alvarez A et al. PillCamColon Capsule for the study of colonic pathology in clinical practice. Study of agreement with colonoscopy. Rev Esp Enferm Dig 2011; 103: 69-75

[17] Spada C, Hassan C, Ingrosso M et al. A new regimen of bowel preparation for PillCam colon capsule endoscopy: a pilot study. Dig Liver Dis 2011; 43: 300-304

[18] Rondonotti E, Borghi C, Mandelli G et al. Accuracy of capsule colonoscopy and computed tomographic colonography in individuals with positive results from the fecal occult blood test. Clin Gastroenterol Hepatol 2014; 12: 1303-1310

[19] Hagel AF, Gabele E, Raithel M et al. Colon capsule endoscopy: detection of colonic polyps compared with conventional colonoscopy and visualization of extracolonic pathologies. Can J Gastroenterol Hepatol 2014; 28: 77-82

[20] Triantafyllou K, Viazis N, Tsibouris P et al. Colon capsule endoscopy is feasible to perform after incomplete colonoscopy and guides further workup in clinical practice. Gastrointest Endosc 2014; 79: 307-316

[21] Saito Y, Saito S, Oka S et al. Evaluation of the clinical efficacy of colon capsule endoscopy in the detection of lesions of the colon: prospective, multicenter, open study. Gastrointest Endosc 2015; 82: 861-869

[22] Rex DK, Adler SN, Aisenberg J et al. Accuracy of capsule colonoscopy in detecting colorectal polyps in a screening population. Gastroenterology 2015; 148: 948-957 e942

[23] Spada C, Hassan C, Barbaro B et al. Colon capsule versus CT colonography in patients with incomplete colonoscopy: a prospective, comparative trial. Gut 2015; 64: 272-281

[24] Togashi K, Fujita T, Utano K et al. Gastrografin as an alternative booster to sodium phosphate in colon capsule endoscopy: safety and efficacy pilot study. Endosc Int Open 2015; 3: E659-E661

[25] Boal Carvalho P, Rosa B, Dias de Castro F et al. PillCam COLON 2 in Crohn's disease: A new concept of pan-enteric mucosal healing assessment. World J Gastroenterol 2015; 21: 7233-7241

[26] Romero-Vazquez J, Caunedo-Alvarez A, Belda-Cuesta A et al. Extracolonic findings with the PillCam Colon: is panendoscopy with capsule endoscopy closer? Endosc Int Open 2016; 4: E1045-E1051

[27] Ota Y, Yamada A, Kobayashi Y et al. Diagnostic capability of colon capsule endoscopy for advanced colorectal cancer: A pilot study. Dig Endosc 2017; 29: 695-701

[28] Zhou J, Tang X, Wang J et al. Feasibility of a novel low-volume and sodium phosphate-free bowel preparation regimen for colon capsule endoscopy. Exp Ther Med 2017; 14: 1739-1743

[29] Nogales O, Garcia-Lledo J, Lujan M et al. Therapeutic impact of colon capsule endoscopy with PillCam COLON 2 after incomplete standard colonoscopy: a Spanish multicenter study. Rev Esp Enferm Dig 2017; 109: 322-327

[30] Igawa A, Oka S, Tanaka S et al. Evaluation for the clinical efficacy of colon capsule endoscopy in the detection of laterally spreading tumors. Digestion 2017; 95: 43-48

[31] Sato J, Nakamura M, Watanabe $\mathrm{O}$ et al. Prospective study of factors important to achieve observation of the entire colon on colon capsule endoscopy. Therap Adv Gastroenterol 2017; 10: 20-31

[32] Pioche M, Ganne C, Gincul R et al. Colon capsule versus computed tomography colonography for colorectal cancer screening in patients with positive fecal occult blood test who refuse colonoscopy: a randomized trial. Endoscopy 2018; 50: 761-769
[33] Takano R, Osawa S, Uotani T et al. Evaluating mucosal healing using colon capsule endoscopy predicts outcome in patients with ulcerative colitis in clinical remission. World J Clin Cases 2018; 6: 952-960

[34] Kobaek-Larsen M, Kroijer R, Dyrvig AK et al. Back-to-back colon capsule endoscopy and optical colonoscopy in colorectal cancer screening individuals. Colorectal Dis 2018; 20: 479-485

[35] Okabayashi S, Kobayashi T, Nakano M et al. A simple 1-day colon capsule endoscopy procedure demonstrated to be a highly acceptable monitoring tool for ulcerative colitis. Inflamm Bowel Dis 2018; 24: 2404-2412

[36] Eliakim R, Spada C, Lapidus A et al. Evaluation of a new pan-enteric video capsule endoscopy system in patients with suspected or established inflammatory bowel disease - feasibility study. Endosc Int Open 2018; 6: E1235-E1246

[37] Parodi A, Vanbiervliet G, Hassan C et al. Colon capsule endoscopy to screen for colorectal neoplasia in those with family histories of colorectal cancer. Gastrointest Endosc 2018; 87: 695-704

[38] Baltes P, Bota M, Albert J et al. PillCamColon2 after incomplete colonoscopy - A prospective multicenter study. World J Gastroenterol 2018; 24: 3556-3566

[39] Voska M, Zavoral M, Grega T et al. Accuracy of colon capsule endoscopy for colorectal neoplasia detection in individuals referred for a screening colonoscopy. Gastroenterol Res Pract 2019: doi:10.1155/ 2019/5975438

[40] Yamada K, Nakamura M, Yamamura T et al. Clinical factors associated with missing colorectal polyp on colon capsule endoscopy. Digestion 2020; 101: 316-322

[41] Gonzalez-Suarez B, Pages M, Araujo IK et al. Colon capsule endoscopy versus CT colonography in FIT-positive colorectal cancer screening subjects: a prospective randomised trial-the VICOCA study. BMC Med 2020; 18: 255

[42] Otani I, Oka S, Tanaka S et al. Diagnostic Yield of colon capsule endoscopy in detection of superficial colorectal lesions. Digestion 2020; 101: 262-269

[43] Pecere S, Senore C, Hassan C et al. Accuracy of colon capsule endoscopy for advanced neoplasia. Gastrointest Endosc 2020; 91: 406-414 e401

[44] Utano K, Katsuki S, Matsuda T et al. Colon capsule endoscopy versus ct colonography in patients with large non-polypoid tumours: a multicentre prospective comparative study (4CN Study). Digestion 2020; 101: 615-623

[45] Eliakim R, Fireman Z, Gralnek IM et al. Evaluation of the PillCam Colon capsule in the detection of colonic pathology: results of the first multicenter, prospective, comparative study. Endoscopy 2006; 38: 963-970

[46] Spada C, Riccioni ME, Hassan C et al. PillCam colon capsule endoscopy: a prospective, randomized trial comparing two regimens of preparation. J Clin Gastroenterol 2011; 45: 119-124

[47] Hartmann D, Keuchel M, Philipper M et al. A pilot study evaluating a new low-volume colon cleansing procedure for capsule colonoscopy. Endoscopy 2012; 44: 482-486

[48] Kakugawa Y, Saito $Y$, Saito $S$ et al. New reduced volume preparation regimen in colon capsule endoscopy. World J Gastroenterol 2012; 18: 2092-2098

[49] Arguelles-Arias F, San-Juan-Acosta M, Belda A et al. Preparations for colon capsule endoscopy. Prospective and randomized comparative study between two preparations for colon capsule endoscopy: PEG 2 liters + ascorbic acid versus PEG 4 liters. Rev Esp Enferm Dig 2014; 106: 312-317

[50] Ramos L, Alarcon O, Adrian Z et al. One-day versus two-day cleansing for colon capsule endoscopy: a prospective randomized pilot study. Gastroenterol Hepatol 2014; 37: 101-106 
[51] Brechmann T, Schmiegel W, Klute L et al. Feasibility of a colon capsule overnight procedure followed by colonoscopy. Z Gastroenterol 2016; 54: 146-151

[52] Kastenberg D, Burch WC Jr., Romeo DP et al. Multicenter, randomized study to optimize bowel preparation for colon capsule endoscopy. World J Gastroenterol 2017; 23: 8615-8625

[53] Alvarez-Urturi C, Fernandez-Esparrach G, Ibanez IA et al. Accuracy of colon capsule endoscopy in detecting colorectal polyps in individuals with familial colorectal cancer: could we avoid colonoscopies? Gastroenterol Res Pract 2017: doi:10.1155/2017/1507914

[54] Buijs MM, Kobaek-Larsen M, Kaalby L et al. Can coffee or chewing gum decrease transit times in Colon capsule endoscopy? A randomized controlled trial BMC Gastroenterol 2018; 18: 95

[55] Kroijer R, Dyrvig AK, Kobaek-Larsen M et al. Booster medication to achieve capsule excretion in colon capsule endoscopy: a randomized controlled trial of three regimens. Endosc Int Open 2018; 6: E1363E1368

[56] Ohmiya N, Hotta N, Mitsufuji S et al. Multicenter feasibility study of bowel preparation with castor oil for colon capsule endoscopy. Dig Endosc 2019; 31: 164-172

[57] Spada C, Hassan C, Galmiche JP et al. Colon capsule endoscopy: European Society of Gastrointestinal Endoscopy (ESGE) Guideline. Endoscopy 2012; 44: 527-536

[58] Lieberman DA, Ghormley J, Flora K. Effect of oral sodium phosphate colon preparation on serum electrolytes in patients with normal serum creatinine. Gastrointest Endosc 1996; 43: 467-469

[59] Hassan C, East J, Radaelli F et al. Bowel preparation for colonoscopy: European Society of Gastrointestinal Endoscopy (ESGE) Guideline Update 2019. Endoscopy 2019; 51: 775-794

[60] Rex DK, Vanner S]. Colon cleansing before colonoscopy: does oral sodium phosphate solution still make sense? Can J Gastroenterol 2009; 23: $210-214$

[61] Schreuders EH, Ruco A, Rabeneck L et al. Colorectal cancer screening: a global overview of existing programmes. Gut 2015; 64: 1637-1649

[62] Koulaouzidis A, Marlicz W, Wenzek $\mathrm{H}$ et al. Returning to digestive endoscopy normality will be slow and must include novelty and telemedicine. Dig Liver Dis 2020; 52: 1099-1101

[63] MacLeod C, Wilson P, Watson AJM. Colon capsule endoscopy: an innovative method for detecting colorectal pathology during the COVID-19 pandemic? Colorectal Dis 2020; 22: 621-624
[64] Buijs MM, Ramezani MH, Herp J et al. Assessment of bowel cleansing quality in colon capsule endoscopy using machine learning: a pilot study. Endosc Int Open 2018; 6: E1044-E1050

[65] Blanes-Vidal V, Baatrup G, Nadimi ES. Addressing priority challenges in the detection and assessment of colorectal polyps from capsule endoscopy and colonoscopy in colorectal cancer screening using machine learning. Acta Oncol 2019; 58: S29-\$36

[66] Spada C, Hassan C, Costamagna G. Colon capsule endoscopy in colorectal cancer screening: a rude awakening from a beautiful dream? Clin Gastroenterol Hepatol 2015; 13: 2302-2304

[67] Spada C, Hassan C, Bellini D et al. Imaging alternatives to colonoscopy: CT colonography and colon capsule. European Society of Gastrointestinal Endoscopy (ESGE) and European Society of Gastrointestinal and Abdominal Radiology (ESGAR) Guideline - Update 2020. Endoscopy 2020: doi:10.1055/a-1258-4819

[68] Cash BD, Fleisher MR, Fern S et al. Multicentre, prospective, randomised study comparing the diagnostic yield of colon capsule endoscopy versus $C T$ colonography in a screening population (the TOPAZ study). Gut 2020: doi:10.1136/gutjnl-2020-322578

[69] Kaminski MF, Thomas-Gibson S, Bugajski M et al. Performance measures for lower gastrointestinal endoscopy: a European Society of Gastrointestinal Endoscopy (ESGE) Quality Improvement Initiative. Endoscopy 2017; 49: 378-397

[70] Rex DK, Schoenfeld PS, Cohen J et al. Quality indicators for colonoscopy. Gastrointest Endosc 2015; 81: 31-53

[71] Zhao S, Wang S, Pan P et al. Magnitude, risk factors, and factors associated with adenoma miss rate of tandem colonoscopy: a systematic review and meta-analysis. Gastroenterology 2019; 156: 1661-1674 e1611

[72] Kaalby L, Deding U, Kobaek-Larsen M et al. Colon capsule endoscopy in colorectal cancer screening: a randomised controlled trial. BM] Open Gastroenterol 2020; 7: doi:10.1136/bmjgast-2020-000411

[73] Singhal S, Nigar S, Paleti V et al. Bowel preparation regimens for colon capsule endoscopy: a review. Therap Adv Gastroenterol 2014; 7: 115122

[74] Adler SN, Hassan C, Metzger Y et al. Second-generation colon capsule endoscopy is feasible in the out-of-clinic setting. Surg Endosc 2014; 28: $570-575$ 